\title{
Geometric Magic Squares
}

\section{Lee Sallows}

This column is a place for those bits of contagious

mathematics that travel from person to person in the

community, because they are so elegant, surprising,

or appealing that one has an urge to pass them on.

Contributions are most welcome.

Please send all submissions to the Mathematical

Entertainments Editor, Ravi Vakil, Stanford University,

Department of Mathematics, Bldg. 380, Stanford,

CA 94305-2125, USA

e-mail: vakil@math.stanford.edu
A magic square is a square array of numbers so arranged that their sum taken in any row, any column, or along either diagonal, is the same. Figure $1 \mathrm{a}$ shows a famous example, the "Lo shu", a $3 \times 3$ specimen of Chinese origin dating from the 4th century BC.

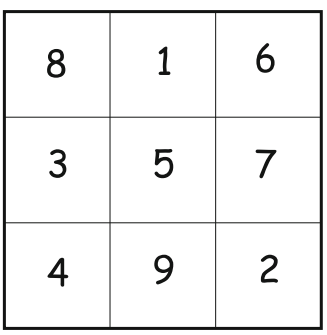

(a)

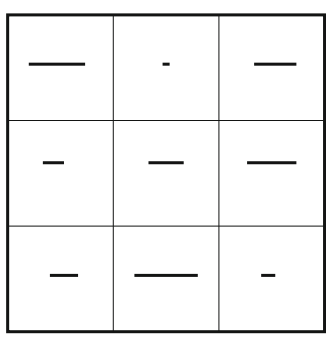

(b)
Figure I. The Lo shu magic square in numerical form (left) and in geometrical form (right).

The topic enjoys an extensive literature: books, articles and websites abound. By now we might expect that virtually every aspect of these curiosities had been exhaustively explored.

Nevertheless, in 2001 I hit upon an innovation that has cast magic squares in an entirely new light. Viewed anew, numerical magic squares are better understood as a special instance of a wider class of geometrical magic squares. Traditional magic squares featuring numbers are then revealed as that particular case of such a "geomagic" square in which the elements are all one-dimensional (1-D), which is to say, they are straight-line segments of a given length.

Consider, for example, a spatial equivalent of the Lo shu seen in Figure 1b, in which line segments of length 1,2,3,.. replace like-valued numbers in each cell. The three lines occupying each row, column, and diagonal can be joined head to tail so as to form or "pave" the same straight line segment of length 15 .

But just as line segments can pave longer line segments, so areas can pave larger areas, volumes can pack roomier volumes, and so on up through higher dimensions. In traditional magic squares we add numbers to form a constant sum, which is to say, we "pave" a one-dimensional space with onedimensional "tiles". What happens beyond the one-dimensional case?

Figure 2 shows a $3 \times 3$ two-dimensional (geo)magic square, its cells occupied by nine distinct planar or 2-D shapes or "pieces". Any three entries in a straight line can be assembled to pave an identically shaped region known as the target, in this case a $6 \times 6$ square, as shown to right and below in the figure. Note how some pieces appear one way in one target, while flipped and/or rotated in another. Thin grid lines on pieces within the square help identify their precise shape and relative size. 


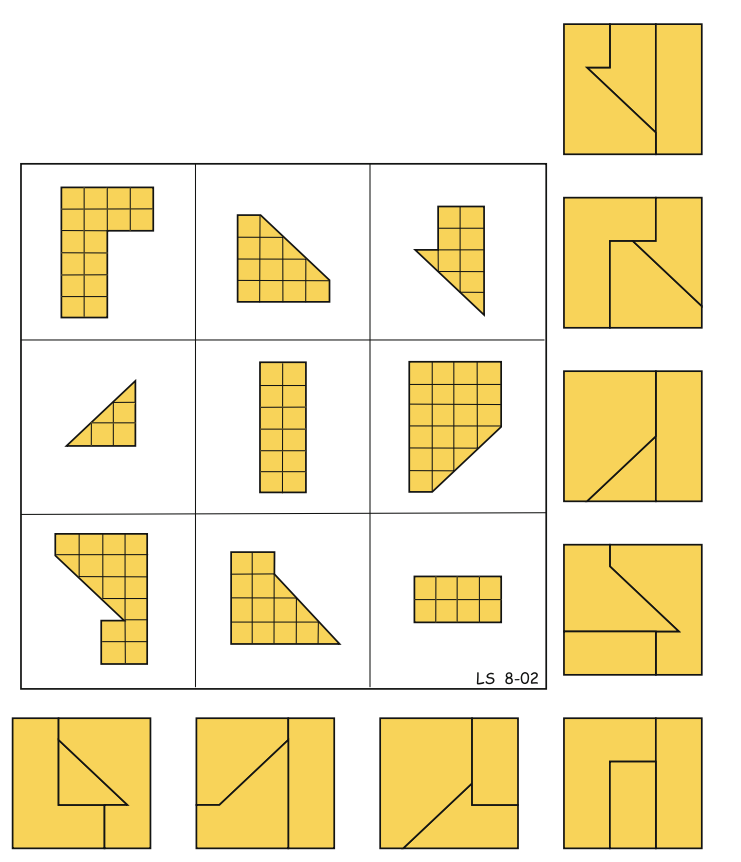

Figure 2. A 2-D geomagic square of order 3 . The 3 pieces in any row, column or diagonal tile the same $6 \times 6$ square target.

Analogously, 3-D magic squares in which solid pieces combine to form a constant 3-D target can also be found. I have one before me as I write, the target of which is a cube; see Figure 3. Likewise geomagic squares using higher dimensional entries also exist, if less easy to visualize. By the dimension of a geomagic square we refer to the dimension of its entries. For a formal definition of geomagic squares, see http://www.GeomagicSquares.com/, which also includes a large gallery of geomagic squares exhibiting a rich variety of special properties.

Staying with 2-D types, an array of $N \times N$ planar pieces is called "magic" when the $N$ entries occurring in each row, column, and both main diagonals, can be fitted together jigsaw-wise to tile an identical region without gaps or overlaps. In tessellating this target, pieces may be rotated or reflected.

Below we shall see that pieces may also be disjoint or disconnected. As with numerical magic squares, geomagics showing repeated entries are deemed trivial. Rotated or

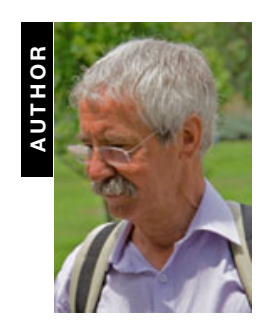

LEE SALLOWS was born in 1944 and was raised in post-war London. He has lived in Nijmegen, The Netherlands, for the past 40 years. Until recently he worked as an electronics engineer for the Radboud University. A handful of published articles on computational wordplay and recreational mathematics are the only fruits of an idle, if occasionally inventive, life.

Johannaweg 12

Nijmegen 6523 MA

The Netherlands

e-mail: lee.sal@inter.nl.net
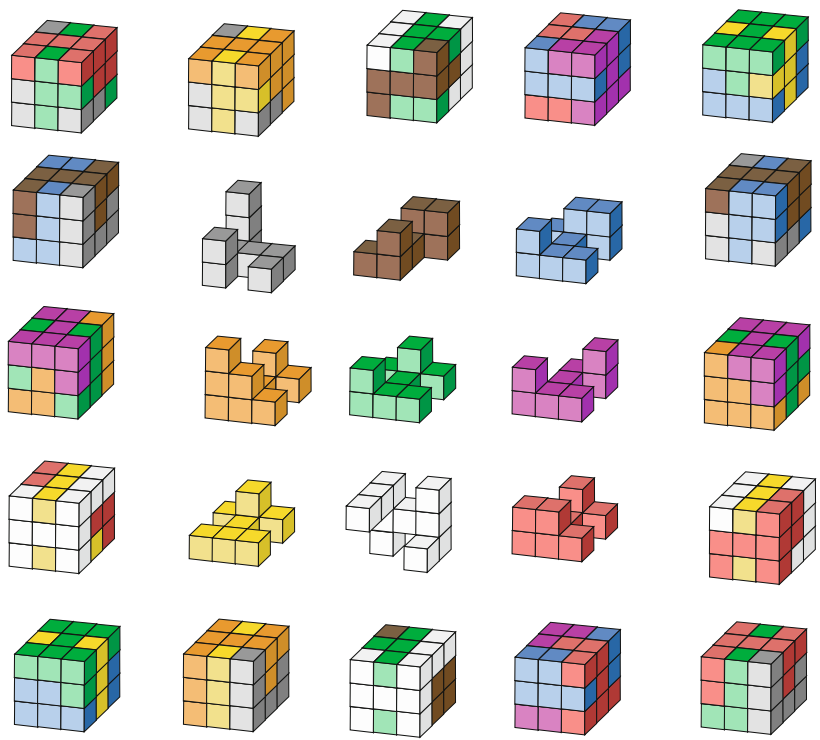

Figure 3. A 3-D geomagic square of order 3 . The 3 pieces in any row, column or diagonal pack the same $3 \times 3 \times 3$ cube.

reflected versions of the same specimen are counted identical. A square of size $N \times N$ is said to be of $\operatorname{order} N$.

As we have seen, every numerical magic square corresponds to a 1-D geometrical magic square written in shorthand notation. But this is not to say that numerical squares account for all possible 1-D geomagic squares. In fact they account only for that subset of 1-D squares using connected line segments. Figure 4 shows a 1-D geomagic square of order 3 that includes disjoint pieces, or pieces composed of two or more separated islands bearing a fixed spatial relation to each other. The overall shape of the compound piece is thus preserved even if moved. Here the 1-D lines have been broadened and coloured to enhance clarity, a trick that could obviously be extended so as to yield a true 2-D geomagic square sporting rectangular targets. However, the point to be made here is that Figure 3 is a 1-D geomagic square for which there exists no corresponding numerical magic square. Magic squares using numbers thus account for no more than a small fraction of all 1-D geomagic squares.

There is a second way to create a geometrical analog of any numerical magic square, which is to use circular arcs or sectors of appropriate angle, rather than straight line segments. Figure 5 shows an example based on the Lo shu. Since the constant sum is 15 , the smallest sector subtends an angle of $360 \div 15=24^{\circ}$. Clearly the target could be replaced by a

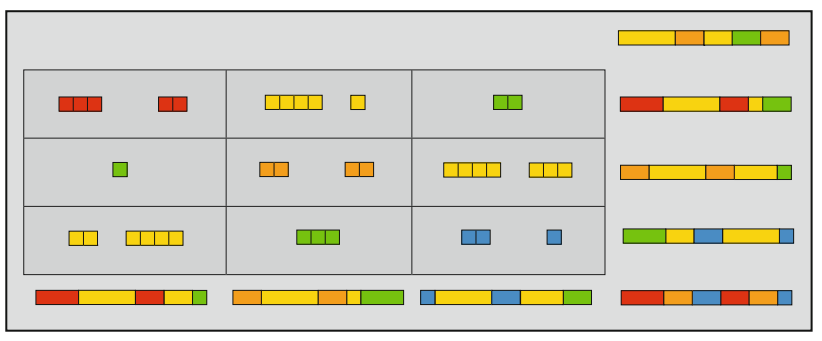

Figure 4. A one-dimensional geomagic square of order 3 using (thickened) disjoint line segments. The target is of length 12 units. 


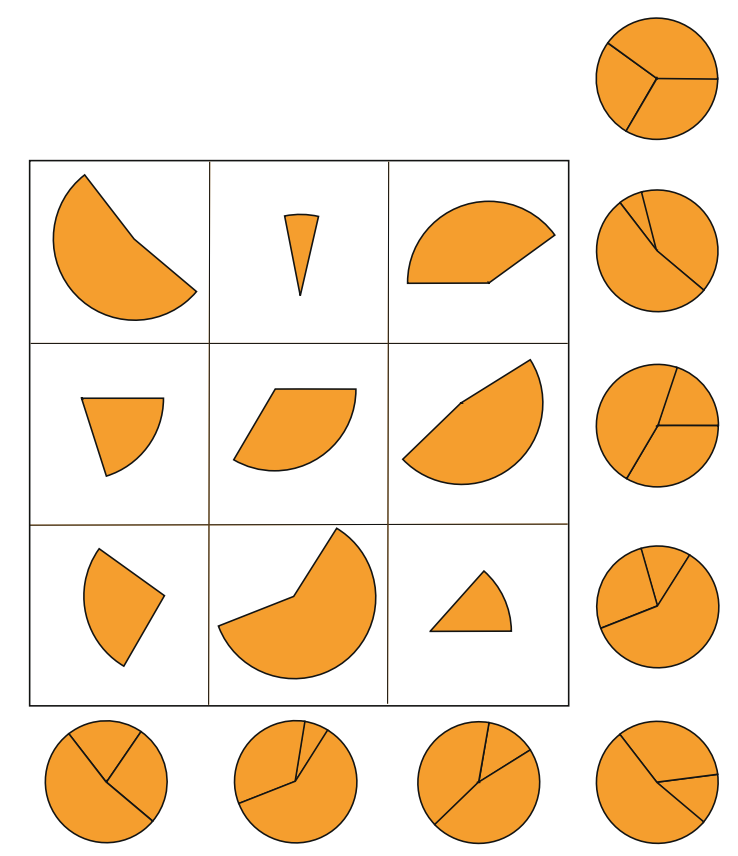

Figure 5. A geometrical version of the Lo shu using circular, rather than linear segments. Here the target is a complete circle but could have been any desired fraction thereof.

regular 15-gon, the sectors then changing to 15-gon segments of corresponding size. Further possible targets may occur to the reader.

As before, circular arc pieces do not have to be connected. Figure 6 shows a $3 \times 3$ square using disjoint arcs, their unit segments here simplified into single coloured dots. Once again, such disconnected pieces cannot be represented by

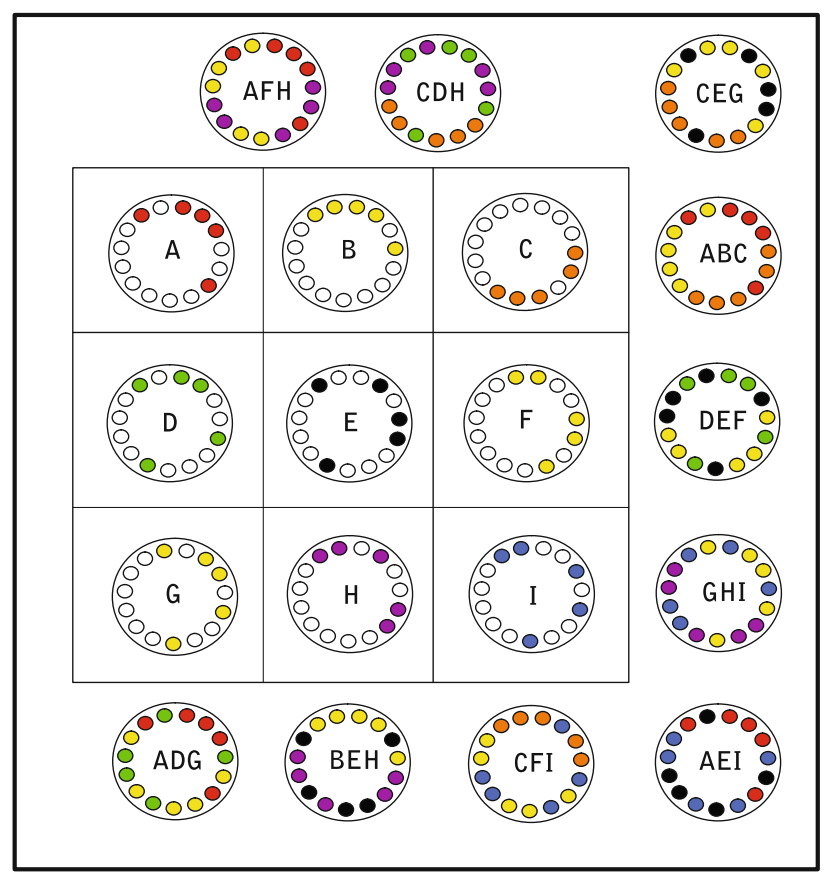

Figure 6. A semi-panmagic square of order 3 using disjoint circular arcs. The latter are represented by unit colored dots of size $360 / 15=24$ degrees.

\begin{tabular}{|c|c|c|}
\hline$c+a$ & $c-a-b$ & $c+b$ \\
\hline$c-a+b$ & $c$ & $c+a-b$ \\
\hline$c-b$ & $c+a+b$ & $c-a$ \\
\hline
\end{tabular}

Figure 7. An algebraic generalization of numerical magic squares of order 3, due to the renowned French mathematician Édouard Lucas [1842-91].

single numbers. But Figure 6 is of greater interest in demonstrating an important, if unsurprising, fact, namely that 2-D geomagic squares listen to laws different from those holding for 1-D types.

As shown by the two targets at top, Figure 6 is a "semipanmagic" square. That is, in addition to rows, columns, and main diagonals, the target is tiled by the "broken" diagonals $\mathrm{AFH}$ and $\mathrm{CDH}$. However, the impossibility of a $3 \times 3$ numerical semi-panmagic square is shown by Figure 7 , which is Lucas' general formula that describes the structure of every $3 \times 3$ numerical magic square.

As with AFH in Figure 6, suppose now that $a, b, c$ in Figure 7 are assigned values such that $(c+a)+(c+a-b)+$ $(c+a+b)=3 c=$ the magic constant. But then $a$ equates to zero, which entails $c+a=c-a=c$, meaning repeated entries. A non-trivial solution therefore does not exist.

It was in fact Lucas's formula that first led to the idea of geomagic squares. Such algebraic formulas had long held for me a peculiar fascination. As I put it in an unpublished essay (Magic Formulae, 1980) on the topic, "Every algebraic square is like an x-ray photograph exposing a skeletal structure underlying the numbers." A vague notion of finding some kind of graphical representation that would make that skeleton visible haunted me for years.

Twenty years later, thinking once again about Lucas's formula, I hit on a new approach. Suppose the three variables in the formula are each represented by a distinct planar shape. Then the entry $c+a$ could be shown as shape $c$ appended to shape $a$, whereas the entry $c-a$ would become shape $c$ from which shape $a$ has been excised. And so on for

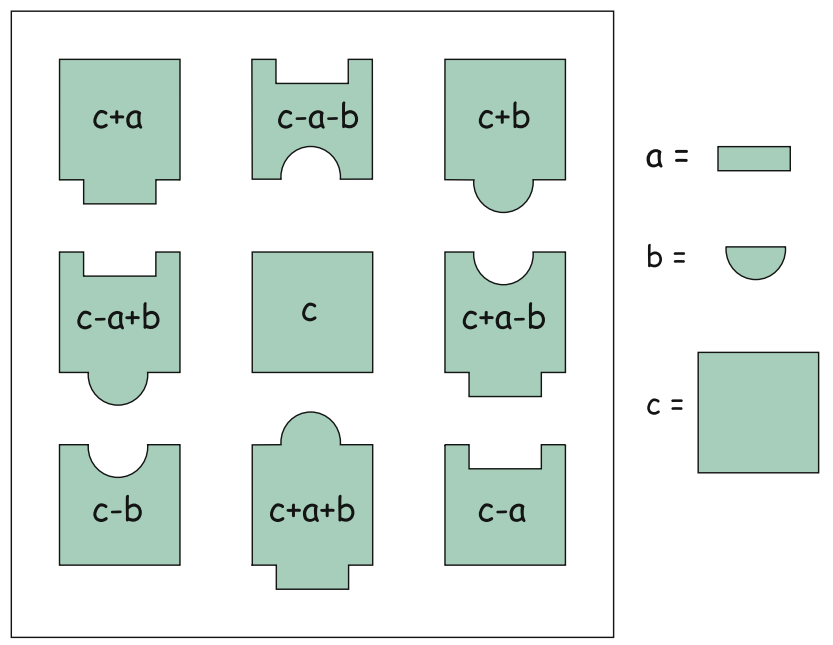

Figure 8. This pictorial representation of Figure 7 first prompted the idea of a geomagic square. 


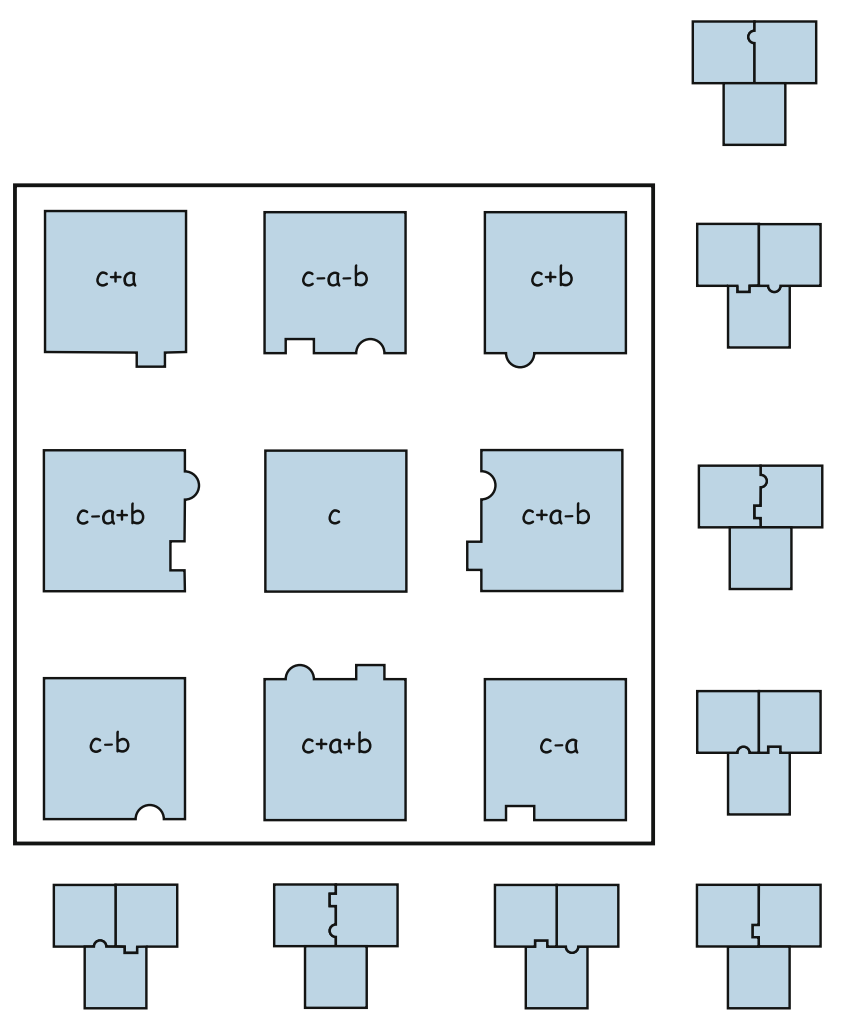

Figure 9. A true geomagic square derived by interpreting Lucas's formula as a template.

the remaining entries. A back-of-the-envelope trial then led to Figure 8 , in which $a$ is a rectangle, $b$ a semi-circle, and $c$ a relatively larger square, three essentially arbitrary choices. This result was more effective than anticipated, the match between keys and keyholes making it easy to imagine the pieces interlocking, and thus visually obvious that the total area of any three in a straight line is the same as a rectangle of size $1 \times 3$, or three times the area of the central piece, in agreement with the formula.

However, the fact that the three central row and three central column pieces do not fit together to complete a rectangle, as the pieces in all other cases will, now seemed a glaring flaw. The desire to find a similar square lacking this defect was then inevitable, and the idea of a geometric magic square was born. Figure 9 shows a second attempt that made good the shortcomings of the first.

Note that, like Figure 9, 5 is itself a geometrical analog of Lucas's formula, the variables $a, b$, and $c$ then corresponding to circular segments of $72^{\circ}, 24^{\circ}$, and $120^{\circ}$, respectively. And the same will go for variants of Figure 5 using alternative targets. However, although it is natural to regard all such trivial variants as essentially the same geomagic square, we should hardly describe Figures 5 and 9 as equivalent, even though they share a common algebraic ancestor. In fact a clear definition of equivalence has thus far proved an elusive quarry, a shortcoming that can sometimes reveal itself in a degree of ambiguity.

The problem of how to go about producing new geometric magic squares now took centre stage. Following much deliberation on this question, two approaches gradually emerged: (1) pencil and paper methods based on algebraic

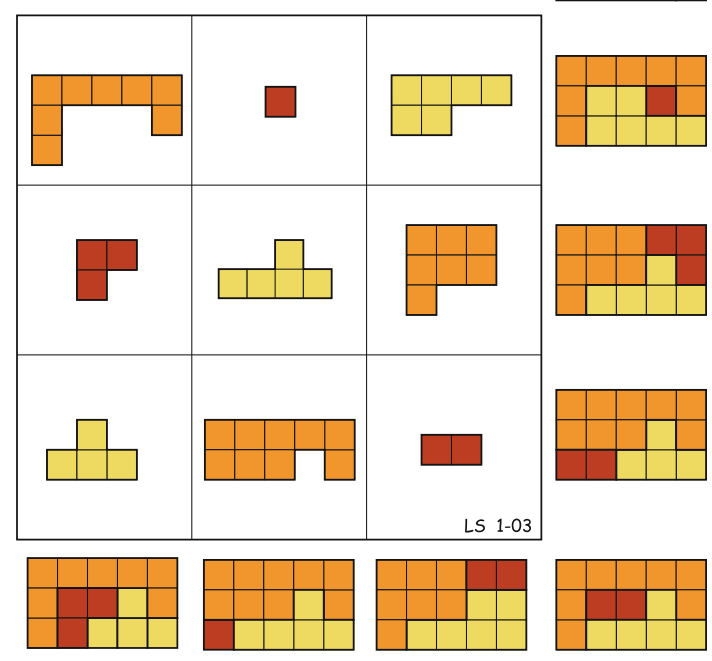

Figure 10. A geomagic square using polyominoes of sizes 1-9. The very existence of such a square had once seemed a daring idea. In reality this is one of 1411 similar solutions, all with a same $3 \times 5$ target.

templates, along the lines just mentioned, (2) in the case of squares restricted to polyforms or shapes built up from repeated atoms, brute force searches by computer. Foremost among the polyforms are polyominos (built up from unit squares), polyiamonds (equilateral triangles) and polyhexes

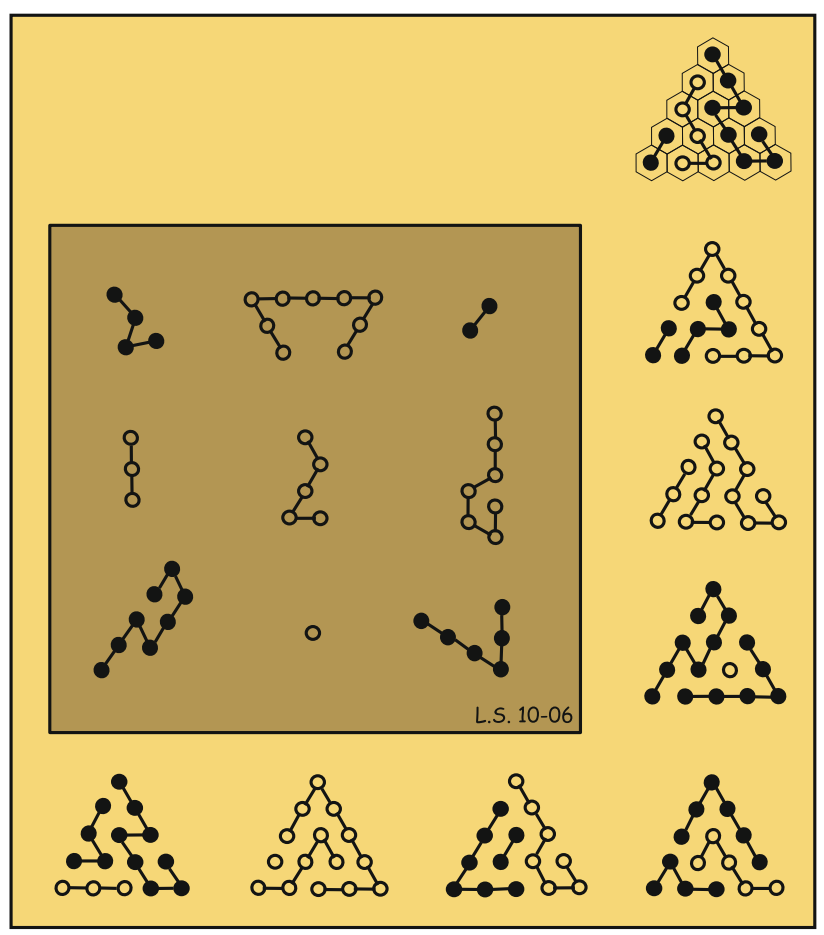

Figure II. A further example of a square using pieces of consecutive size, in this case polyhexes. Here the latter have been reduced to dots and lines to produce a diagram reminiscent of a well known Chinese rendering of the Lo shu. 

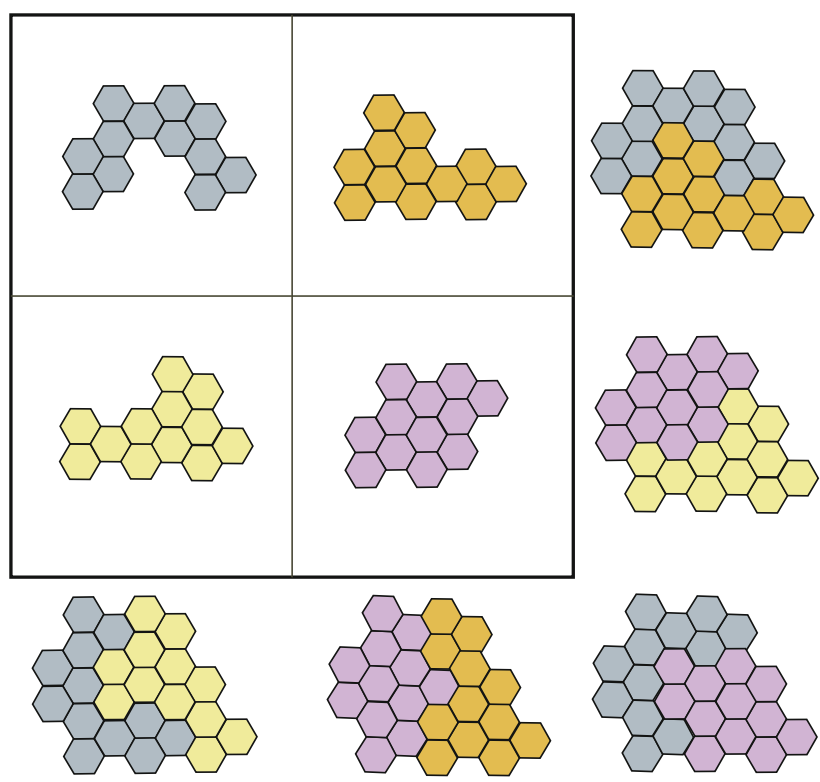

Figure 12. A near miss at a geomagic square of order 2. The question of whether or not there exists a fully magic solution occupied me for years.

(regular hexagons). On the aforementioned website I present a selection of some of the more interesting squares brought to light by these two methods.

In most cases, the examples shown have been discovered in response to some pertinent question, such as: Does there exist a $3 \times 3$ square composed of nine polyominos with sizes in consecutive order? As a trophy-hunter, I found the prospect of getting such an exotic gem enticing. Often such questions entailed weeks of work before arriving at an answer. In this case, the outcome shattered every expectation. Figure 10 presents one of the 1411 different solutions, target in each case being a $3 \times 5$ rectangle. And if this prolixity was surprising, what to make of Figure 11, which is among 169,344 alternatives, the all using 9 polyhexes of the same size [here reduced to nodes linked by lines] and the same target?

With a single exception, the 2-D squares to follow are all of size $3 \times 3$ or $4 \times 4$, larger squares being to my mind of scant interest. It is a common fallacy that the bigger the square the greater the achievement, because of the supposed difficulty of getting so many numbers to comply with the magic conditions. On the contrary, the constraints implied diminish rapidly with increasing size, as is shown by the algebraic generalization of the $N \times N$ numerical magic square, which can be written so that it contains $N^{2}-2 N$ cells each containing a single free variable.

Turning to the other end of the scale, clearly a magic square of size $2 \times 2$ cannot be realized using four distinct numbers. The smallest numerical magic squares are thus of order 3, and the same is true of "semi-magic" squares, which are those that are magic on rows and columns only. However, Figure 12 shows a non-trivial $2 \times 2$ semi-magic square using 2-D pieces. It is based on a finding due to Michael Reid. Note that besides rows and columns, one diagonal is magic.

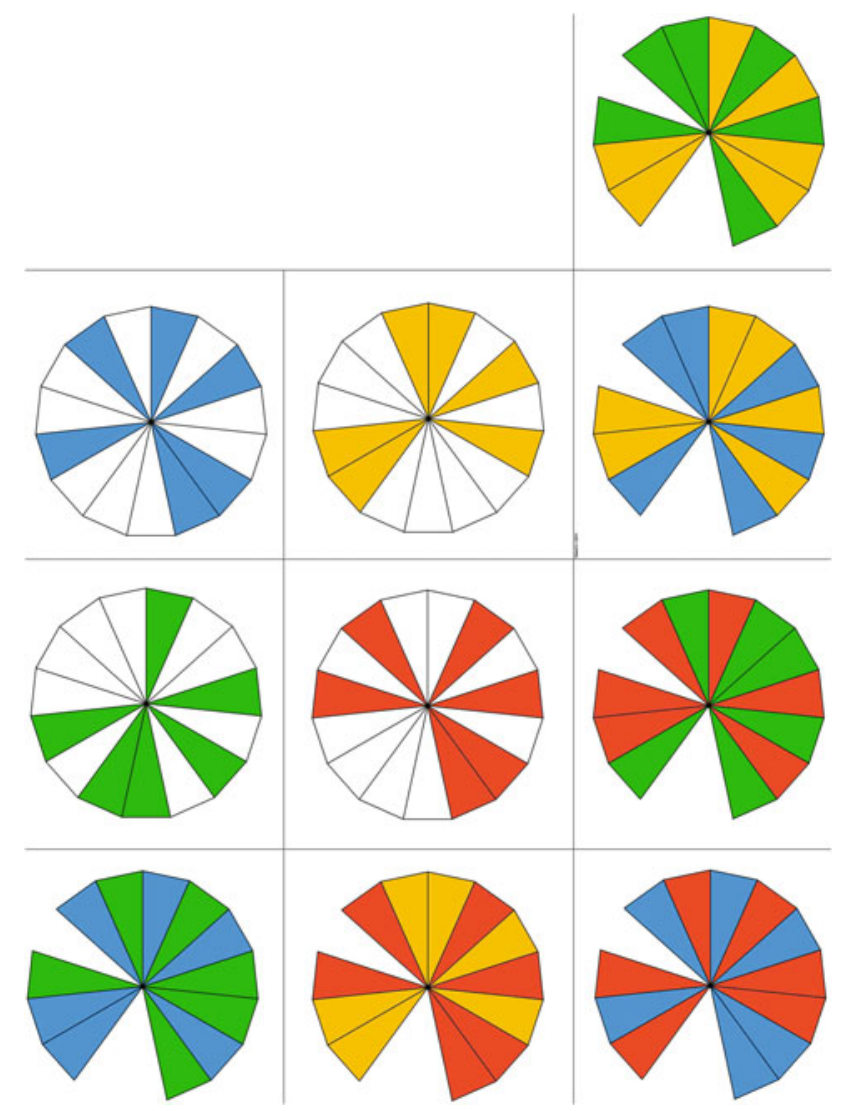

Figure 13. The first ever $2 \times 2$ geomagic square due to Frank Tinkelenberg of the The Netherlands. The square uses disconnected pieces and a disconnected target. Does there exist a solution using connected pieces? The question remains unanswered.

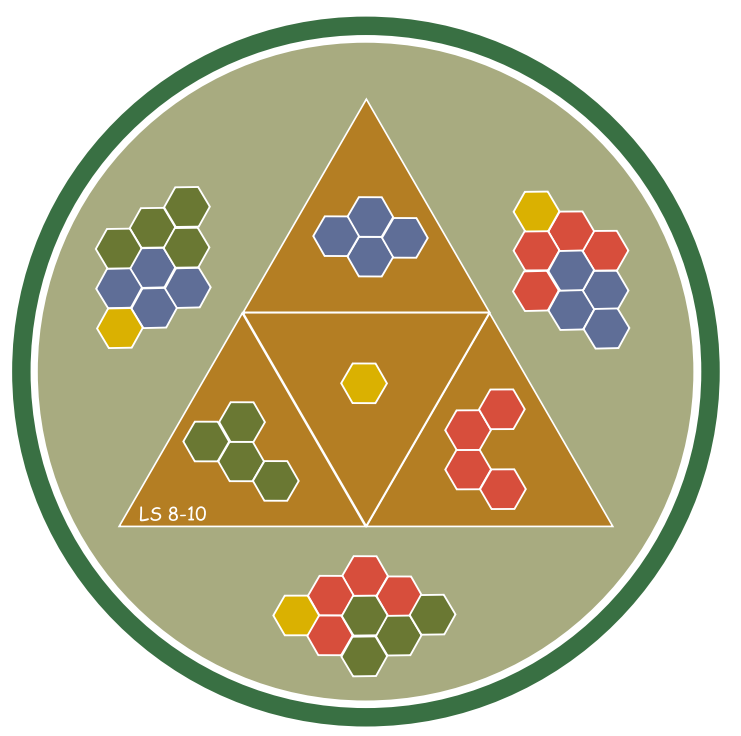

Figure 14. A deceptively simple-seeming geomagic triangle. The discovery of such specimens is a lot harder than first sight suggests. 


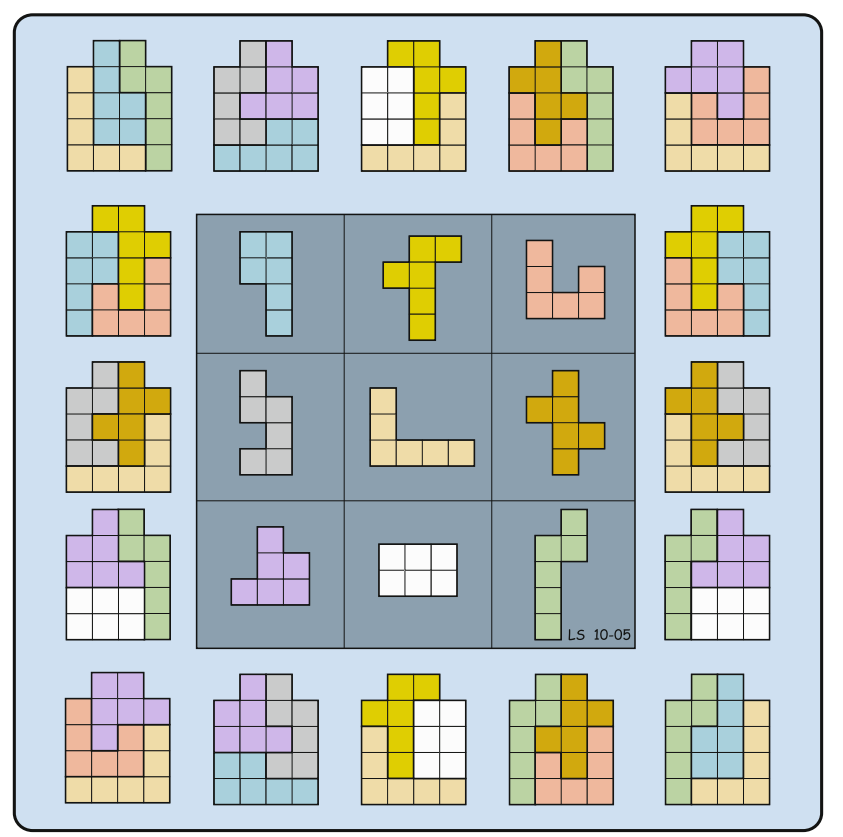

Figure 15. Order 3 geomagic squares using pieces of the same area are far rarer (and thus more difficult to find) than those using unequal pieces. This one uses nine hexominoes. In searching for such specialities different target shapes must be tried. The result in this case was felicitous.

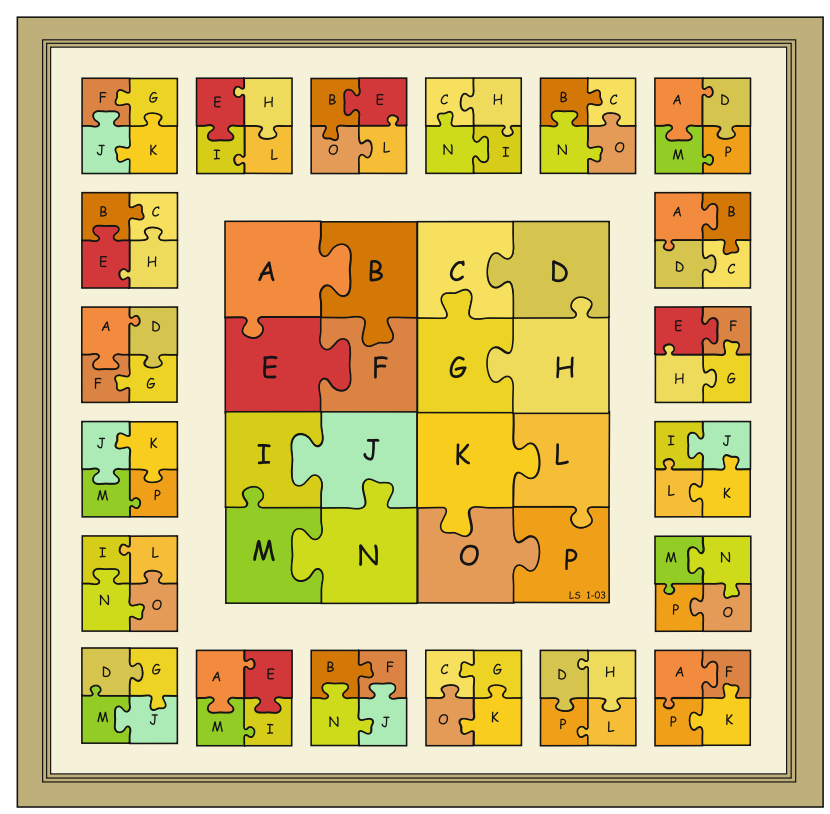

Figure 16. This magic jigsaw puzzle is an example of what I call a "self-interlocking" geomagic square. The 16 pieces are no longer separated from each other within their cells, but interlock so as to pave a single square area. I had never imagined that such a structure was possible until an examination of the geometrical analogues of certain algebraic magic squares forced their existence upon me. The visual harmony of the square is a reflection of the symmetries to be found in the algebraic magic square on which it is based.

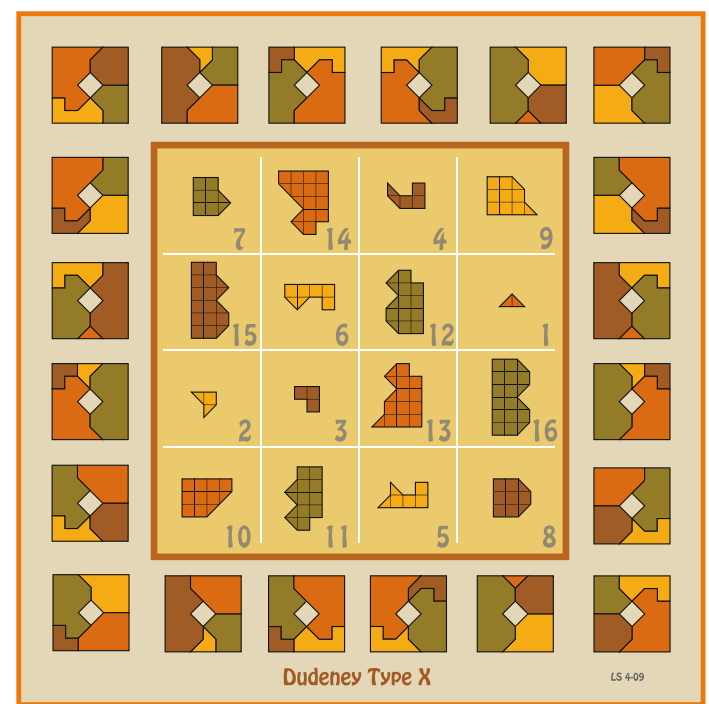

Figure I7. Here the title, Dudeney Type $\mathrm{X}$, is a reference to $\mathrm{H}$. E. Dudeney, the famous British counterpart to America's Sam Loyd, and author of many wonderful puzzle books during the late 19th and early 20th centuries. Dudeney's original work on numerical magic squares included a classification of the 880 normal squares into 12 types, depending upon how their complementary pairs, 1 and 16, 2 and 15, etc., were distributed. In his system, the above square is of Type X, or type ten.

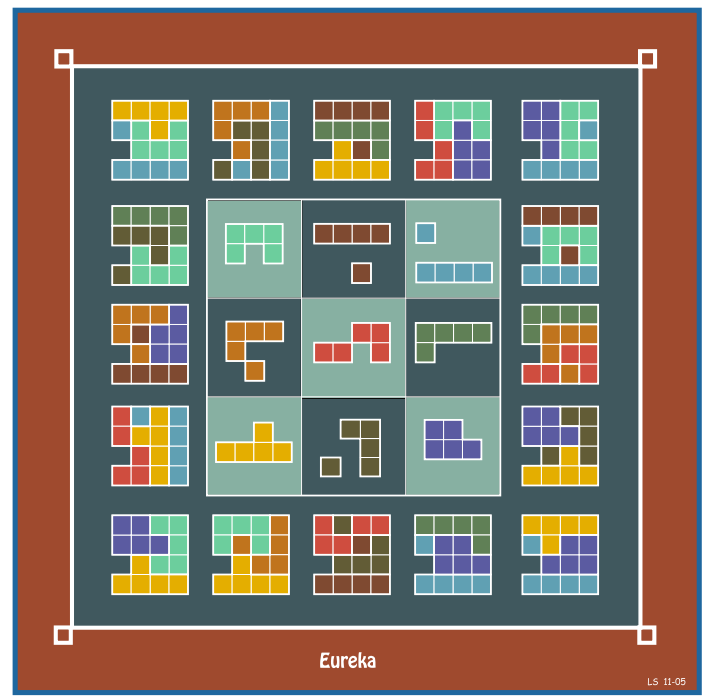

Figure 18. A $3 \times 3$ panmagic or nasik square, which is one in which every diagonal, broken or otherwise, is magic. In this case, the target can also be formed by any three of the four corner pieces. This square was of particular interest to me because, in the realm of numerical magic squares, panmagics of $3 \times 3$ are impossible. The possibility of finding 2-D panmagics of $3 \times 3$ was thus exciting and their initial discovery an event to celebrate. The resort to disjoint pieces is an indication of the difficulty encountered in finding it. Such nuggets are thin on the ground. In an as yet unpublished paper I prove that the nine entries in any 2 -D panmagic $3 \times 3$ square can always be rearranged to yield 54 distinct panmagic squares, rotations and reflections not counted. 


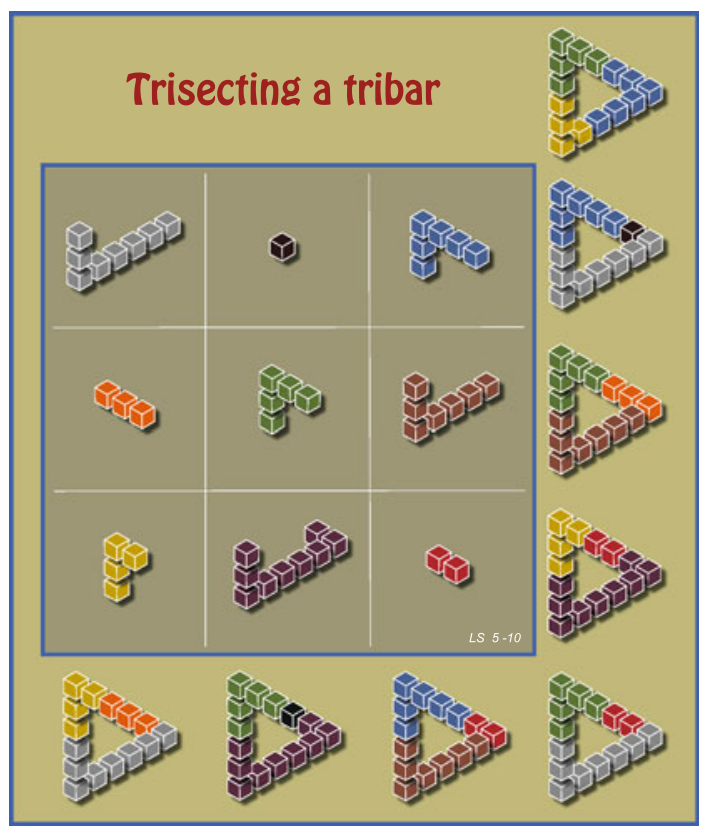

Figure 19. The target here is a variation on the first ever impossible figure that depicted a similar "object", but using 9 rather than 15 cubes. This was invented by Oscar Reutersvard in 1934. Later, in 1958, Penrose and Penrose, unaware of Reutersvard's work, published an equivalent figure composed of three solid beams, nowadays known as the Penrose tribar. The above is one of two solutions using the same target and similar "pieces" of size 1,2,... . The idea of such a target had occurred to me long ago. Following countless failed attempts, I finally found a way to do it, eight years later.

Until very recently, every attempt to discover a fully magic 2-D square of order 2 had failed. However, following an airing of the problem with the recent launch of my website GeomagicSquares.com, Frank Tinkelenberg, a Dutch software developer, finally cracked the problem with a square using disconnected pieces and a disconnected target; see Figure 13. In any case, the extreme difficuly met with in tracking down this solution is merely further confirmation of the point just made, that the smaller the square, the greater the constraints, or the fewer the degrees of freedom. The question now remaining is whether or not there exists a $2 \times 2$ square using connected pieces? Meanwhile, a slightly related device can be seen in the magic triangle of Figure 14.

Figure 3 illustrated the 3-D square with cubic target referred to earlier. With a little patience the precise shapes of

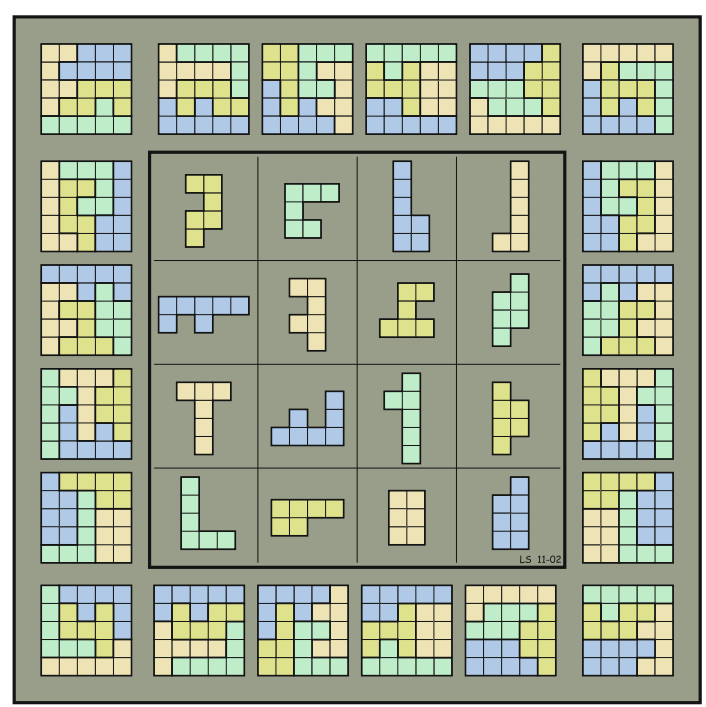

Figure 20. A computer-discovered $4 \times 4$ geomagic square. Every magic line contains three hexominoes and one heptomino. $3 \times 6+7=5 \times 5$, the area of the square target.

the pieces can be inferred, although the deficiencies of trying to present these and higher-dimensional specimens via the page will be apparent. Two dimensional squares, on the other hand, present no such difficulty, being not only almost self-explanatory, but both elegant and ornamental besides. Hence my focus on 2-D squares in the present article.

Although I am no artist, in creating pictures I have taken pains to present each square to the best effect. But appearances should not distract. Fundamentally, every square is a timeless Platonic form, a constellation in the firmament of logical space consisting in a nexus of geometrical relations. The latter are of no particular significance perhaps. But for all that, they remain among the immutable and eternal patterns woven into the magic carpet of mathematics.

The geomagic squares below are taken from the Gallery of Lee Sallows's website http://www.GeomagicSquares.com/, which includes a wealth of further examples, among them Figures 15-20, here reproduced.

\section{OPEN ACCESS}

This article is distributed under the terms of the Creative Commons Attribution Noncommercial License which permits any noncommercial use, distribution, and reproduction in any medium, provided the original author(s) and source are credited. 